\title{
Cortical Selectivity through Random Connectivity
}

\author{
Joe Corey and Benjamin Scholl \\ Institute of Neuroscience, The University of Texas at Austin, Austin, Texas 78712 \\ Review of Hansel and van Vreeswijk
}

In visual cortex (V1), neurons are highly organized for many different stimulus properties including orientation selectivity, retinotopy, and ocular dominance. Since the discovery of functional architecture in monkeys (Powell and Mountcastle, 1959) and the detailed examination of orientation columns in cat (Hubel and Wiesel, 1962), it has been thought that V1 organization is essential for extracting sensory information and generating highly selective responses in neurons. Surprisingly, however, this functional architecture has not been found in some visual animals, particularly rodents. In the visual cortex of rats, mice, and the gray squirrel there is no evidence for orientation maps or cortical columns, although there still exists highly orientation-selective neurons (Ohki and Reid, 2007). Instead, extracellular recordings and two-photon calcium imaging have revealed a salt-and-pepper organization of orientation selectivity (Ohki and Reid, 2007).

Visual cortex organization appears to diverge among mammals, with primates and carnivores possessing a regular columnar architecture, while rodents show no obvious structure, despite all having neurons strongly selective for oriented visual stimuli. This species difference raises questions about the role of columnar organization in information processing

Received May 22, 2012; revised June 12, 2012; accepted June 14, 2012. We are grateful to Nicholas Priebe, Andrew Tan, and David Hansel for helpful discussions.

Correspondence should be addressed to Benjamin Scholl, Institute of Neuroscience, The University of Texas at Austin, 2400 Speedway, Austin, TX 78712. E-mail: scholl.ben@gmail.com.

DOI:10.1523/JNEUROSCI.2463-12.2012

Copyright $\odot 2012$ the authors $\quad 0270-6474 / 12 / 3210103-02 \$ 15.00 / 0$ and sensory computation. For example, does the emergence of orientation selectivity require organized cortical connectivity or are random connections sufficient? Hansel and van Vreeswijk (2012) sought to determine whether robust orientation tuning in animals without columnar organization can arise merely from random connectivity. To address this issue, the authors constructed a network model of visual cortex and demonstrated that strong orientation selectivity can emerge from random connectivity (Fig. 1).

Envisioning cortical cell layers as twodimensional sheets, the authors defined connection probability between neurons as a function of cortical distance and not similarity in stimulus preference. This neural network has a feedforward design, such that synaptic inputs from one neuron sheet (layer 4) converge onto neurons in a second (layer 2/3; Fig. 1). Since neurons in layer 4 have a salt-and-pepper organization of orientation selectivity, neurons in layer $2 / 3$ receive a random mixture of inputs (Fig. 1). There is also recurrent input in layer $2 / 3$, with connection probabilities defined only as a function of cortical distance and not functional similarity. Using a realistic conductance-based cell model and a network comprised of both excitatory and inhibitory neurons, Hansel and van Vreeswijk (2012) demonstrated that a cortical network without any functional structure, either between neuron layers or within a layer, can still generate orientation selectivity (Hansel and van Vreeswijk, 2012, their Fig. 4).

This remarkable finding can be explained by considering the orientation tuning of individual inputs. Since orientation tuning of synaptic inputs onto layer $2 / 3$ are random, the ensemble tuning is reduced through simple averaging as the number of inputs increase. This creates a very large untuned component and a relatively nonexistent tuned component. Because this network is populated by both excitatory and inhibitory neurons operating in a balanced activity regime, untuned excitatory and inhibitory inputs roughly cancel each other (Hansel and van Vreeswijk, 2012, their Fig. 2). This cancellation causes the remaining tuned components to dominate responses and render neurons strongly orientation selective. Interestingly, Hansel and van Vreeswijk (2012) found that the emergence of orientation selectivity is largely independent to the degree of orientation selectivity in the input layer. In fact, in agreement with empirical evidence showing that stimulus orientation and spatial frequency preference is roughly equivalent across laminar layers (Niell and Stryker, 2008), differences in the degree of selectivity between layer 4 and layer $2 / 3$ were either small or nonexistent (Hansel and van Vreeswijk, 2012, their Table 2). They also show that abolishing tuning of layer 4 inhibitory neurons does not drastically affect the emergence of orientation selectivity, which is important considering that inhibitory neurons are more broadly tuned than excitatory neurons in mouse visual cortex (Kerlin et al., 2010). These findings show that orientation selectivity can be generated through random synaptic inputs, with local connection probabilities determined by cortical distance, suggesting a nonspecific synap- 
tic mechanism which rodents might use to extract visual stimulus features.

Although their model exhibits aspects of rodent visual cortex, Hansel and van Vreeswijk (2012) do not make any claims about this being the sole mechanism in generating orientation selectivity. Random connections might be sufficient, but this does not imply that feature-selective functional connectivity is absent in animals with "salt-and-pepper" organization. It is possible that the orientation selectivity arising from random connections is further strengthened by precise fine-scale local connectivity and that these two systems work in tandem (Fig. 1). For example, it has been shown that there exists fine-scale organization in intracortical connections between neighboring neurons in mouse visual cortex, where nearby neurons with similar orientation preference have a higher probability of being connected to one another (Ko et al., 2011). Given this knowledge, Hansel and van Vreeswijk (2012) could modify their recurrent network component and weight connection probabilities between neurons by both cortical distance and stimulus preference. For example, increasing the connection probability of nearby neurons with similar orientation preference might further enhance their selectivity (Fig. 1).

It should be mentioned that functional connectivity arising purely from random connections is unlikely when considering synaptic plasticity. During spike timing-dependent plasticity, for example, synaptic connections between neurons of similar orientation preference can be strengthened by repeated stimulus presentations. A synergistic resolution to random versus specific connectivity might be found when considering experience-dependent plasticity during development. Before the critical period, mice are exposed to relatively little visual stimulation, yet orientation selectivity exists (Wang et al., 2010). Random connectivity may initially

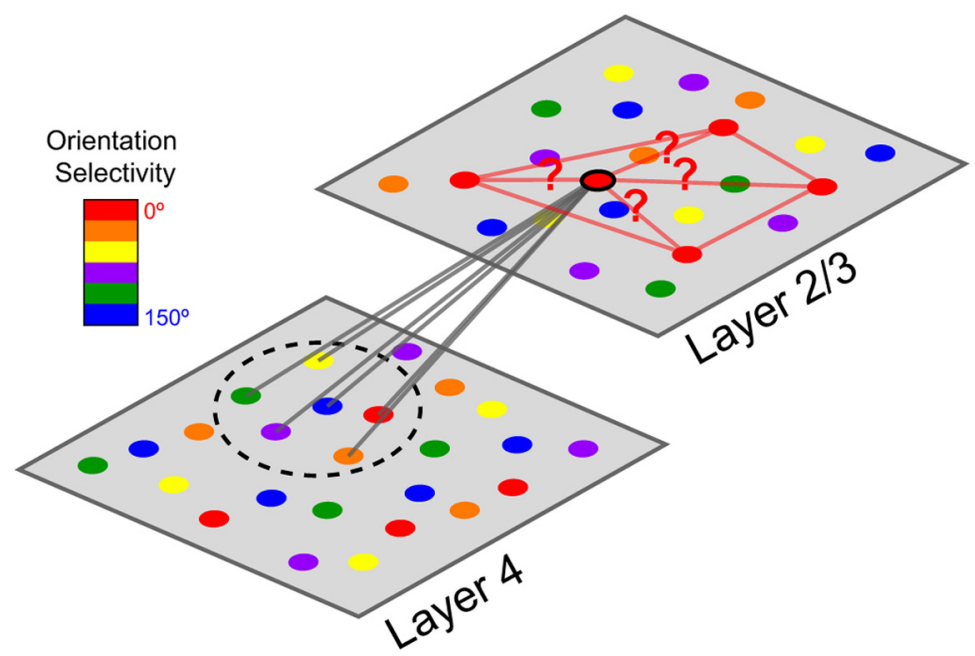

Figure 1. Schematic of network model from Hansel and Vreeswijk (2012). Orientation selectivity in layer 4 is distributed randomly in a salt-and-pepper fashion. Selectivity in layer 2/3 emerges through random connections (gray lines). Fine-scale connections (red lines) are not necessary for emergent tuning, but might enhance selectivity in tandem.

provide a scaffolding for orientation tuning, which is improved upon during development. This suggests a developmental progression whereby random connections generate selectivity that is later shaped by learning mechanisms in the form of featureselective plasticity.

These authors have shown that robust orientation tuning in animals without a functional map can arise merely from random connectivity, dislodging the notion that functional organization of neuron response properties is required to generate orientation selectivity. In addition, this emergent selectivity was largely independent of the input orientation selectivity and constraints placed on inhibitory neurons. In the future, it will be important to establish the role of random synaptic connections in generating stimulus feature selectivities, particularly during development, whereby these types of synaptic mechanisms could create the framework of cortical organization and a basis for strengthening selectivity through experience-dependent plasticity.

\section{References}

Hansel D, van Vreeswijk C (2012) The mechanism of orientation selectivity in primary visual cortex without a functional map. J Neurosci 32:4049-4064.

Hubel DH, Wiesel TN (1962) Receptive fields, binocular interaction and functional architecture in the cat's visual cortex. J Physiol Lond 160:106-154.

Kerlin AM, Andermann ML, Berezovskii VK, Reid RC (2010) Broadly tuned response properties of diverse inhibitory neuron subtypes in mouse visual cortex. Neuron 67: $858-871$

Ko H, Hofer SB, Pichler B, Buchanan KA, Sjöström PJ, Mrsic-Flogel TD (2011) Functional specificity of local synaptic connections in neocortical networks. Nature 473: 87-91.

Niell CM, Stryker MP (2008) Highly selective receptive fields in mouse visual cortex. J Neurosci 28:7520-7536.

Ohki K, Reid RC (2007) Specificity and randomness in the visual cortex. Curr Opin Neurobiol 17:401-407.

Powell TP, Mountcastle VB (1959) Some aspects of the functional organization of the cortex of the postcentral gyrus of the monkey: a correlation of findings obtained in a single unit analysis with cytoarchitecture. Bull Johns Hopkins Hosp 105:133-162.

Wang BS, Sarnaik R, Cang J (2010) Critical period matches binocular orientation preference in the visual Cortex. Neuron 65:246256. 